\title{
Application of Power System Short-Term Load Forecasting Based on Wavelet Analysis \\ linsuo Zeng ${ }^{1,}$ a, Xinmiao Jia ${ }^{1, b}$, Jiafeng $\mathrm{Xu}^{2,}$,, Yuliang Jia ${ }^{2, b}$ \\ ${ }^{1}$ School of Shenyang University of Technology, Shengyang, 110000, China \\ ${ }^{2}$ Chaoyang Power Supply Company, Chaoyang, 122000, China \\ azls1004@hotmail.com , bmm2260@163.com
}

Keywords: power load forecasting, wavelet analysis, wavelet neural network

\begin{abstract}
Power load forecasting is an important part of power grid scheduling,operation and maintenance.It is also the basis of economic operation and security of electric power system.It has important significance to the stability and development of electric power system.This paper introduces the theory of wavelet analysis,and combined with neural network to form a wavelet neural network forecasting model,analysis the characteristics of the wavelet neural network,wavelet neural network algorithm design.Comparison of the results of the wavelet neural network and BP neural network,it is proved that the wavelet neural network model has the characteristics of high prediction accuracy,fast convergence speed,better prediction effect.
\end{abstract}

\section{Introduction}

Short-term load forecasting is an important task of the electricity sector,it is to ensure the reliability and security of the power system,and improve the power grid of the economy from the overall level has a significant role in the planning,electric power planning and scheduling must be based on load forecasting ${ }^{[1]}$.

\section{Short term load forecasting based on wavelet analysis}

\subsection{Wavelet analysis}

Wavelet analysis is a mathematical method for signal analysis. Wavelet analysis method has been applied to many branches, such as functional analysis,numerical analysis,Fourier analysis ${ }^{[2]}$,spline analysis,harmonic analysis, and so on.The main idea is to choose the suitable wavelet basis function $\phi(t)$, and then to the generating function of wavelet basis function $\left\{\phi_{a, b}\right\}$ :

$$
\phi_{a, b}(t)=|a|^{-\frac{1}{2}} \phi\left(\frac{t-b}{a}\right)
$$

And then through the analysis of basis function $\left\{\phi_{a, b}\right\}$.The same as Fourier analysis,wavelet analysis has many kinds,such as continuous wavelet transform,discrete wavelet transform,fast wavelet transform,fast wavelet transform and it is also called wavelet multi-resolution analysis,etc.For a function $f(t)$, the continuous wavelet transform is defined as:

$$
W_{f}(a, b) \leq f, \phi_{a, b} \geq|a|^{-\frac{1}{2}} \int_{R} f(t) \phi\left(\frac{t-b}{a}\right) d t
$$

The $\mathrm{b}$ represents the displacement of time,the a represents the time scale, $W_{f}(a, b)$ referring to the original signal $f(t)$ in the b space contains the wavelet function of a,which indicates the degree of correlation between the signal $f(t)$ and the wavelet function $\phi\left(\frac{t-a}{b}\right)$. The corresponding inverse transformation equations for continuous wavelet transform is: 


$$
f(t)=\frac{1}{C} \iint_{R^{2}} W_{f}(a, b) \phi_{a, b}(x) \frac{d a}{a^{2}} d b
$$

In the type, $C_{\phi}$ satisfies the formula (2-4)

$$
\iint_{R^{2}} W_{f}(a, b) \bar{W}_{f}(a, b) \frac{d a}{a^{2}} d b=C_{\phi}
$$

The wavelet transform and the commonly Fourier transform can be used to do frequency domain analysis.But Fourier transform can only get the signal frequency domain information in the whole time domain.If the signals superimposed $g(t)$ at a given time $t_{0}$, the volatility $f(t)$ will generate an increase $\Delta F(w)$ in the Fourier transform $F(w)$ of the signal,But we can not judge by the frequency domain information leading $F(w)$ to a generation of time-domain component of the definition of domain $g(t)$,don't know when has $g(t)$ produce and its duration.In order to analyze the signal in the time domain,it can be used for short-time Fourier transform.The method is to apply a sliding window $w(t-\tau)$ (the $\tau$ reflects the position of the sliding window), and then conduct the Fourier transformation.When the $\mathrm{w}$ changes, the width and shape of the sliding window do not change.And the shape of the time frequency window of wavelet transform can be adjusted.When the scale a is larger,the time-frequency window time domain part of the wider,and analysis of low frequency,suitable for details.This step by step from coarse and fine for analyzing things called as multi-resolution analysis,wavelet transform is an important aspect of engineering application ${ }^{[3],[4]}$.By multi-resolution analysis to analyze the load sequence, its characteristics of different frequency component,thus easy to reveal the regularity of load sequence,and get a more accurate load forecasting model ${ }^{[5]}$.

\subsection{Wavelet neural network}

Wavelet neural network is a new kind of neural network model based on wavelet analysis.It inherits the advantages of wavelet analysis and neural network,so it has better performance.At present,we can use two methods to realize the combination of wavelet analysis and neural network, which are discrete wavelet neural network and continuous wavelet neural network,the former is the first to use wavelet transform to pre-processing in advance,to extract the feature vector and then by the neural network to deal with them,shown in figure 1.The latter is wavelet function instead of hidden layer in the neural network,as shown in figure 2,the training of the network and the learning methods still adopts the traditional neural network's thought ${ }^{[6],[7]}$, so that both the wavelet transform and neural networks will organically combine the advantages.

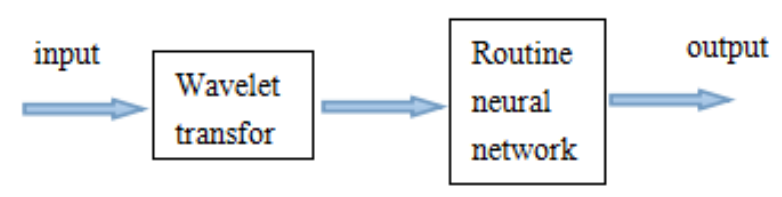

Fig.1 discrete wavelet neural network

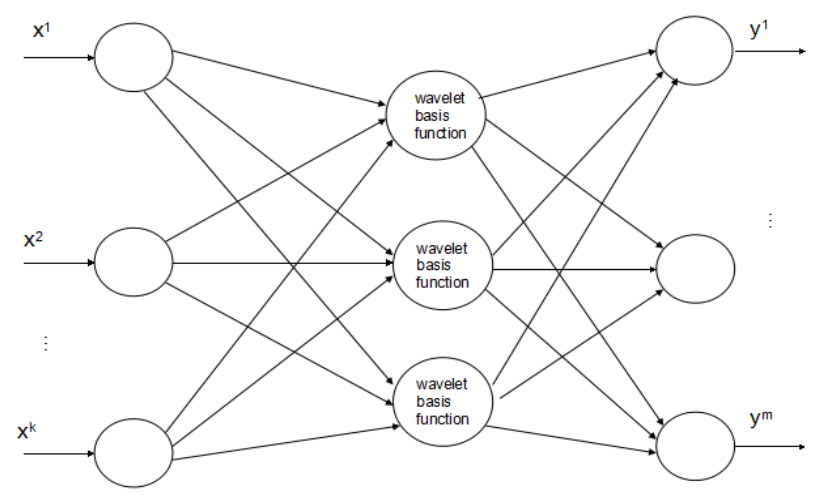

Fig.2 Wavelet neural network 


\subsection{The design of Wavelet neural network algorithm}

Through the analysis of wavelet analysis and wavelet neural network,the wavelet neural network has great advantages in the practical application.Power system short-term load forecasting model for nonlinear load component can be used in forecast analysis on wavelet neural network algorithm $^{[8],[9]}$,In order to ensure the greatest degree of convergence of speed and accuracy.

At the figure $2, X_{1}, X_{2}, \ldots, X_{k}$ represent the input parameters of the wavelet neural network, $Y_{1}, Y_{1}, \ldots, Y_{m}$ represent wavelet neural network prediction output, $w_{i j}$ and $w_{j k}$ are wavelet neural network weights.

The input signal sequence is $x_{i}=1,2, \ldots, k$, The formula for calculating the implied layer output is:

$$
h(j)=h_{j}\left(\frac{\sum_{i=1}^{k} w_{i j} x_{i}-b_{j}}{a_{j}}\right), j=1,2, \ldots, l
$$

In the type, $h(j)$ :output values for the hidden layer of the number $\mathrm{j}$ node; $w_{i j}$ :connection weights for the input layer and the hidden layer; $h_{j}$ :wavelet base function; $b_{j}$ :translation factor of basis function $h_{j} ; a_{j}$ :the scaling factor of the wavelet base function $h_{j}$.

The wavelet basis function used the Morlet wavelet base function,and its mathematical formula is:

$$
y=\cos (1.75 x) e^{\frac{-x^{2}}{2}}
$$

The formula for calculating output layer of wavelet neural network:

$$
y(k)=\sum_{i=1}^{l} w_{i k} h(i), k=1,2, \ldots, m
$$

In the type, $w_{i k}$ :hidden layer to output layer weights; $h(i)$ :the output of the number i hidden layer node; 1 :hidden layer node number;m:output layer node number.

Wavelet neural network algorithm flow chart,Fig.3.

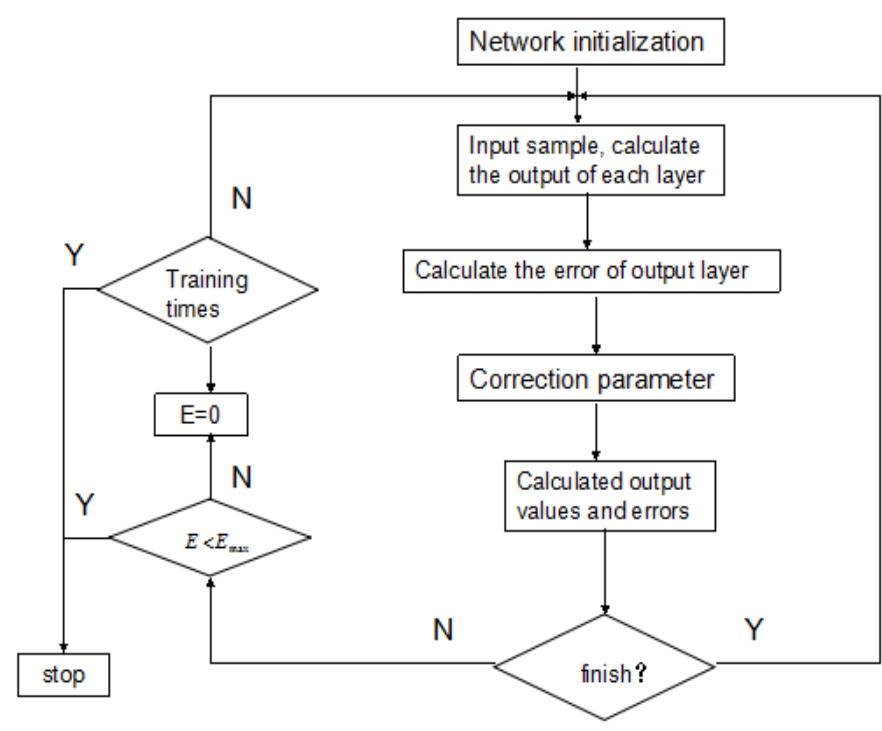

Fig.3 wavelet neural network algorithm flow chart

\section{Examples and results of short term load forecasting}

In this paper,based on the May 10-15,2015 load data of a certain area of Liaoning province,on the basis of forecast on May 20 load data,through comparing the BP neural network and wavelet neural network.

In this paper,BP neural network input layer is used as the 3 nodes,the hidden layer is 6 nodes,the output layer is 1 nodes,the system error is set to 0.0001 , and the maximum training times is 3000 
times.The neural network is trained in the training process, and the training speed: $\eta=0.8$,momentum factor: $\alpha=0.8$.

After the training of BP neural network,the prediction results are compared with the actual values,as shown in table 1.

Table 1 comparison between predictive value of BP neural network and actual value

\begin{tabular}{|c|c|c|c|}
\hline time & prediction load & $\begin{array}{c}\text { actual } \\
\text { load }\end{array}$ & error \\
\hline $0: 00$ & 0.2389 & 0.2124 & 0.1247 \\
\hline $2: 00$ & 0.0542 & 0.0894 & -0.3937 \\
\hline $4: 00$ & 0.1072 & 0.1142 & -0.0612 \\
\hline $6: 00$ & 0.2455 & 0.2359 & 0.0406 \\
\hline $8: 00$ & 0.6512 & 0.5579 & 0.1672 \\
\hline $10: 00$ & 0.6734 & 0.5889 & 0.1434 \\
\hline $12: 00$ & 0.6812 & 0.7042 & -0.0326 \\
\hline $14: 00$ & 0.6990 & 0.7150 & -0.0223 \\
\hline $16: 00$ & 0.7230 & 0.7209 & 0.0029 \\
\hline $18: 00$ & 0.7597 & 0.7462 & 0.0180 \\
\hline $20: 00$ & 0.7964 & 0.8032 & -0.0084 \\
\hline $22: 00$ & 0.8001 & 0.8136 & -0.0165 \\
\hline
\end{tabular}

At the same time,the load forecasting is using by wavelet neural network method.In the wavelet neural network,the system error and the maximum training times are the same as that of BP neural network, which is determined by the wavelet neural network, and there is no of the neurons ${ }^{[10]}$ in the BP neural network.After wavelet neural network training,the prediction results are compared with the actual values, as shown in table 2.

Table 2 Comparison between prediction of wavelet neural network and actual value

\begin{tabular}{|c|c|c|c|}
\hline time & prediction load & $\begin{array}{c}\text { actual } \\
\text { load }\end{array}$ & error \\
\hline $0: 00$ & 0.2259 & 0.2124 & 0.0635 \\
\hline $2: 00$ & 0.0632 & 0.0894 & -0.2930 \\
\hline $4: 00$ & 0.1098 & 0.1142 & -0.0385 \\
\hline $6: 00$ & 0.2256 & 0.2359 & -0.0436 \\
\hline $8: 00$ & 0.6072 & 0.5579 & 0.0883 \\
\hline $10: 00$ & 0.6272 & 0.5889 & 0.0650 \\
\hline $12: 00$ & 0.6901 & 0.7042 & -0.0200 \\
\hline $14: 00$ & 0.6993 & 0.7150 & -0.0219 \\
\hline $16: 00$ & 0.7229 & 0.7209 & 0.0027 \\
\hline $18: 00$ & 0.7560 & 0.7462 & 0.0131 \\
\hline $20: 00$ & 0.7975 & 0.8032 & -0.0070 \\
\hline $22: 00$ & 0.8108 & 0.8136 & -0.0034 \\
\hline
\end{tabular}

Through the two tables,the prediction error of the two methods can be obtained,The average error of the wavelet neural network is 0.016,and the average error of BP neural network load forecasting is 0.031 .Thus,the accuracy of wavelet neural network is higher than that of BP neural 
network,and prediction of wavelet neural network is faster than BP neural network.In general,the prediction method of wavelet neural network is more superior than that of BP neural network.

\section{Summary}

Power system short-term load forecasting is the basis of the normal operation of the power grid,which has important significance to improve the economic benefit of the electric power sector.In order to promote the safe and economical operation of power system,this paper is mainly based on wavelet analysis and optimization of power system short-term load forecasting research.Combining wavelet transform and neural network,research on wavelet neural network algorithm,comparison of two methods of wavelet neural network and BP neural network,the wavelet network has the high accuracy.The wavelet neural network model has the ability of fast convergence and stronger training and learning ability,which has a positive effect on short-term load forecasting.

\section{References}

[1].Chongqing Kang,Qing Xia,Boming Zhang.Research on power system load forecasting and its development direction.Automation of electric power system.2004,28(17):1-3.

[2].MichengLi,JianshuLuo.Waveletanalysis and its application[M].Beijing:Electronics Industry Press,2003,p.11-18.

[3].FushengYang,Wavelet transform analysis and application[M],Science press,2001,p.42-51.

[4].Lee K Y,Cha Y T,Park JH.Short-term load forecasting using an artificial neural network[J].IEEE Transactions on Power Systems,1992,7(1):p.124-132.

[5].Yun Sun,Zhiqiang Li,Guozhong Wu.Power system short-term load forecasting application method of selecting wavelet functions[J].Journal of Jiangnan University,2007,6(1),p.36-38.

[6].Daiyuan Zhang.New theory and method of neural network.Beijing:Tsinghua University press,2006,p.4-18.

[7].Dahai Zhang,Shifang Jiang,Yanqiu Bi.Power load forecasting method based on Wavelet Neural Network.Electric power automation equipment,2003,23(8),p.30-31.

[8].LIU K.Comparison of very short-term load forecasting techniques.IEEE Trans.on Power Systems, 1996,11(2):877-882.

[9].S.J.Kiartzis(s),C.E Zoumas,J.B.Theocharis,etc,Short-term Load Forecasting in an Autonomous Power System Using Artificial Neural Networks.IEEE transaction on Power Systems, Vol,12,No.4,November 1997.

[10].Yu zhao,Yu zhang,Chunjie Qi.Prediction Model of Stock Market Returns Based on Wavelet Neural Network.IEEE Pacific-Asia Workshop on Computational Intelligence and Industrial Application,2008:p.31-36 